OPEN ACCESS

Edited by:

Fernando Antunes,

University of Lisbon, Portugal

Reviewed by:

Jan Kaslin,

Australian Regenerative Medicine

Institute, Australia

Carla Lucini,

University of Naples Federico II, Italy

${ }^{*}$ Correspondence:

Eva Terzibasi Tozzin

eva.terzibasi@szn.it

Specialty section:

This article was submitted to

Cellular Biochemistry,

a section of the journal

Frontiers in Cell and Developmental

Biology

Received: 12 December 2020 Accepted: 26 May 2021

Published: 09 July 2021

Citation:

Bagnoli S and Terzibasi Tozzini E (2021) Age-Dependent Regulation of Notch Family Members in the Neuronal Stem Cell Niches of the Short-Lived Killifish

Nothobranchius furzeri.

Front. Cell Dev. Biol. 9:640958. doi: 10.3389/fcell.2021.640958

\section{Age-Dependent Regulation of Notch Family Members in the Neuronal Stem Cell Niches of the Short-Lived Killifish Nothobranchius furzeri}

\author{
Sara Bagnoli ${ }^{1}$ and Eva Terzibasi Tozzini ${ }^{2 *}$ \\ ${ }^{1}$ Laboratory of Biology (BIO@SNS), Scuola Normale Superiore, Pisa, Italy, ${ }^{2}$ Department of Biology and Evolution of Marine \\ Organisms (BEOM), Stazione Zoologica Anton Dohrn, Naples, Italy
}

Background: The annual killifish Nothobranchius furzeri is a new experimental model organism in biology, since it represents the vertebrate species with the shortest captive life span and also shows the fastest maturation and senescence recorded in the laboratory. Here, we use this model to investigate the age-dependent decay of neurogenesis in the telencephalon (brain region sharing the same embryonic origin with the mammalian adult niches), focusing on the expression of the Notch pathway genes.

Results: We observed that the major ligands/receptors of the pathway showed a negative correlation with age, indicating age-dependent downregulation of the Notch pathway. Moreover, expression of notch1a was clearly limited to active neurogenic niches and declined during aging, without changing its regional patterning. Expression of notch3 is not visibly influenced by aging.

Conclusion: Both expression pattern and regulation differ between notch1a and notch3, with the former being limited to mitotically active regions and reduced by aging and the latter being present in all cells with a neurogenic potential, regardless of the level of their actual mitotic activity, and so is less influenced by age. This finally suggests a possible differential role of the two receptors in the regulation of the niche proliferative potential throughout the entire fish life.

Keywords: notch pathway, ageing, killifish, adult neurogenesis, in situ hybridization, RNA sequencing

\section{INTRODUCTION}

In the last decade, our group has intensively studied the annual fish Nothobranchius furzeri as a new experimental model in biology. N. furzeri has gained recognition as an animal model for aging studies, and currently, it is extensively studied in an increasing number of laboratories worldwide (Kabiljo et al., 2019; Matsui et al., 2019; Montesano et al., 2019; Fumagalli et al., 2020; Hu et al., 2020; Kelmer et al., 2020; Wang et al., 2020). These fish inhabit ephemeral pools in the semiarid bushveld of Southern Mozambique characterized by scarce and erratic precipitations and have adapted to the seasonal drying of their environment by producing desiccation-resistant eggs that can remain dormant in the dry mud for 1 and maybe 
more years by entering into a diapause state (Genade et al., 2005; Cellerino et al., 2016; Kim et al., 2016). Due to very short duration of the rainy season, the natural life span of these animals is limited to a maximum of a few months (Terzibasi Tozzini et al., 2013; Dorn et al., 2014; Vrtílek et al., 2018). They represent the vertebrate species with the shortest captive life span and also the fastest maturation (Genade et al., 2005; Blazek et al., 2013). In addition, they express a series of conserved aging markers and are amenable to genetic manipulations, making them an attractive model system for aging research (Valenzano et al., 2006, 2011; Terzibasi et al., 2007; Hartmann et al., 2009, 2011; Reichwald et al., 2009; Di Cicco et al., 2011; Hartmann and Englert, 2012; Tozzini et al., 2012; Allard et al., 2013), as reviewed in Cellerino et al. (2016) and Kim et al. (2016). The recent sequencing of the N. furzeri genome (Benayoun et al., 2015; Reichwald et al., 2015; Valenzano et al., 2015) and the establishment of transgenic techniques for this species (Valenzano et al., 2011; Hartmann and Englert, 2012; Benayoun et al., 2015; Harel et al., 2015; Ripa et al., 2016; Dolfi et al., 2019) made $N$. furzeri a new versatile model organism to investigate the genetic basis of aging (Platzer and Englert, 2016; Cui et al., 2020). A striking characteristic of killifish development is the presence of diapause, a period when embryonic development is suspended at specific stages (Furness et al., 2015; Hand et al., 2016), such as gastrulation (diapause 1), mid-somitogenesis (diapause 2), and pre-hatching (diapause 3). A surprising result that recently emerged is that the transcriptional patterns observed during aging and diapause overlap to a significant extent (Reichwald et al., 2015), providing the motivation to study development-related genes in the context of aging.

Neurogenesis is the process that generates the nervous system during embryonic development. In mammals, neurogenesis is completed during perinatal life and is restricted to specific telencephalic niches during adult life (Ming and Song, 2011; Urbán and Guillemot, 2014), but in fish brains, adult neurogenesis remains active, with stem cell niches distributed along the entire rostro-caudal extent of the ventricular surface (Zupanc and Horschke, 1995; Adolf et al., 2006; Grandel et al., 2006; Kuroyanagi et al., 2010). Adult neurogenesis in mammals is known to decrease dramatically with age (Kuhn et al., 1996; Pekcec et al., 2008; Ben Abdallah et al., 2010; Knoth et al., 2010), and although the level of adult neurogenesis remains higher compared to mammals, a decrease of this phenomenon has been observed during aging in zebrafish as well (Edelmann et al., 2013; Kaslin et al., 2013; Stankiewicz et al., 2017). We therefore studied adult neuronal stem cells (NSCs) of $N$. furzeri in vivo and demonstrated an age-dependent decay in adult neurogenesis in terms of both incorporation of nucleotide analogs and expression of specific markers (Tozzini et al., 2012). In addition, RNA sequencing (RNA-seq) experiments revealed age-dependent downregulation of neurogenesis genes during aging of N. furzeri brain and identified novel markers of adult NSCs (Baumgart et al., 2014).

Even though fish neurogenic niches are more widespread than the mammalian homologous structures, the telencephalic niches share the same embryonic origin with the mammalian adult niches (Adolf et al., 2006). In particular, the ventral niche is homologous to the mammalian subventricular (subpallial) zone, whereas the dorsolateral region is homologous to the pallial zone (hippocampal mammalian niche). We also analyzed the germinal zone of the optic tectum that is very active in teleosts and whose stem cells retain a neuroepithelial phenotype (Tozzini et al., 2012). This would allow us to differentiate between conserved expression patterns in the telencephalic niches from possible teleost-specific expression patterns detectable only in the optic tectum.

We concentrated our attention on the Notch pathway during aging of the NSCs. The Notch pathway is a key pathway for the control of neurogenesis and cell fate during both embryonic development (Yoon and Gaiano, 2005; Pierfelice et al., 2011; McIntosh et al., 2017; Kanungo et al., 2018) and adult neurogenesis (Suh et al., 2009; Dozawa et al., 2014; Than-Trong et al., 2018; Sueda and Kageyama, 2020). When activated by the ligands (Delta and Jagged), the Notch intracellular domain is cleaved and activates the transcription of effectors of the hairy family (her and hes genes in vertebrates) that act as repressors of gene transcription that block neurogenic genes (Yeo et al., 2007; van Tetering and Vooijs, 2011). Expression of notch genes is retained in adult NSCs where Notch activity can be detected by the activity of the hes 5 promoter (Lugert et al., 2010). In zebrafish, notch family is composed of four members: notch1a, notch1b, notch2, and notch 3 (Bierkamp and Campos-Ortega, 1993; Westin and Lardelli, 1997). Studies in adult zebrafish have revealed a differential activity of notch1 and notch3. Inactivation of notch 3 induces NSC amplification by reactivating them from a quiescent status, promoting symmetric divisions. On the other hand, abrogation of notch 1 function induces new neuron generation, inducing a reduction of the number of activated NSCs (Alunni et al., 2013; Than-Trong et al., 2018). Effects of aging on the Notch pathway remain poorly investigated. We reanalyzed RNA-seq and proteomics data to specifically assess the age-dependent expression of the components of the Notch pathway, and we studied the expression of notch 1 and notch 3 using in situ hybridization (ISH).

\section{MATERIALS AND METHODS}

\section{Fish Breeding and Housing Conditions}

All experiments were performed on group-house $N$. furzeri of the MZM-04/10 strain. The protocols of fish maintenance were carried out in accordance with all animal use practices approved by the Italian Ministry of Health (Number 96/2003a). Ethynyl deoxyuridine (EdU)-injected samples used for the experiments were derived from archived material of the animal stock as previously used by Tozzini et al. (2012).

Eggs were maintained on wet peat moss at room temperature in sealed Petri dishes. When embryos had developed, eggs were hatched by flushing the peat with tap water at $16-18^{\circ} \mathrm{C}$. Embryos were scooped with a cut plastic pipette and transferred to a clean 
vessel. Fry were fed with newly hatched Artemia nauplii for the first 2 weeks and then weaned with finely chopped Chironomus larvae. Starting at the fourth week of life, fish were moved to 40-L tanks at a maximum density of 20 fish per tank equipped with air-driven sponge filters. The aquarium room's temperature was set at a constant $26^{\circ} \mathrm{C}$. Twice a week, the bottom of the tanks was siphoned and $50 \%$ of the water was exchanged with tempered tap water.

\section{In vivo Ethynyl Deoxyuridine Injection}

To localize the neurogenic niches in the brain, fishes were treated with a single intraperitoneal injection of the modified thymidine analog EdU (5-ethynyl-2'-deoxyuridine), which is specifically incorporated in the DNA during the S-phase of proliferating cells. To do that, the Click-iT ${ }^{\circledR}$ EdU Alexa Fluor ${ }^{\circledR} 488$ Imaging Kit from Invitrogen-Thermo Fisher Scientific (cat. no.: C10337) was used: fish were first shortly anesthetized by using a sedation dosage of tricaine methanesulfonate (MS-222), and $50 \mu \mathrm{l}$ of a $10-\mu \mathrm{M}$ EdU solution was injected intraperitoneally in the experimental fish. Finally, animals were euthanized with MS-222 $4 \mathrm{~h}$ after injection and immediately dissected. Preparation and use of MS222 and all procedures conform to the prescription of European (Directive 2010/63/UE) and Italian laws (DL 26/04-03-2014).

\section{Brain Tissue Collection and Preparation}

Whole brains from five young (7 weeks) and five old (25 weeks) animals (listed in Table 1) were dissected and fixed by immersion in $4 \%$ paraformaldehyde/0.1 $\mathrm{M}$ phosphate buffer ( $\mathrm{pH} 7.4$ ) and then cryoprotected with a two-step immersion at $20 \%$ and then $30 \%$ sucrose solution for at least $12 \mathrm{~h}$ each. Finally, the tissues were embedded at $-20^{\circ} \mathrm{C}$ in $\mathrm{Neg} 50$ cryo-embedding medium (Thermo Scientific); series of $16-\mu \mathrm{m}$-thick sections were cut with a Leica cryostat and collected on Superfrost plus slides ${ }^{\circledR}$ (Thermo Scientific).

\section{In situ Hybridization and Ethynyl Deoxyuridine Staining In situ Hybridization}

All ISH protocols have been performed on $16-\mu \mathrm{m}$-thick cryosections of fish brain. Slides were dried for $2 \mathrm{~h}$ at $37^{\circ} \mathrm{C}$, washed in phosphate buffered saline (PBS) twice for $3 \mathrm{~min}$, and then treated for $8 \mathrm{~min}$ with Proteinase K (diluted 1:80,000 starting from stocks of $20 \mathrm{mg} / \mathrm{ml})$. After that, slides were washed in glycine $(2 \mathrm{mg} / \mathrm{ml}$ in PBT solution $=$ PBS + tween $200.1 \%)$ twice for $5 \mathrm{~min}$ to stop the reaction. Then, sections were fixed with paraformaldehyde (PFA) $4 \%$ for $20 \mathrm{~min}$ at room temperature and washed in PBT (three times for $3 \mathrm{~min}$ ). Prehybridization was performed by covering the slides with $200 \mu \mathrm{l}$ of hybridization buffer under parafilm coverslips (to avoid evaporation) at hybridization temperature $\left(60^{\circ} \mathrm{C}\right)$ for $30 \mathrm{~min}$ inside a wet chamber. Hybridization was performed covering each slide with a solution of the specific antisense 3'DIGlabeled probe diluted in $500 \mu \mathrm{l}$ of hybridization buffer to a final concentration of $1 \mu \mathrm{g} / \mathrm{ml}$. Parafilm coverslips were used, and slides were incubated at hybridization temperature overnight. Before using them, diluted RNA probes have been denatured for
TABLE 1 | Total list of experimental animals used in the study.

\begin{tabular}{llccc}
\hline Protocol & $\begin{array}{l}\text { Fish species and } \\
\text { strain }\end{array}$ & $\begin{array}{c}\text { Age } \\
\text { (weeks) }\end{array}$ & $\begin{array}{l}\text { Animals } \\
\text { number }\end{array}$ & N Tot \\
\hline RNA Seq & Species: & 5 & 5 & 25 \\
Data set 1 & Nothobranchius & & & \\
Baumgart et al., 2014 & furzeri & & &
\end{tabular}

Strain: MZM04/10

\begin{tabular}{|c|c|c|c|c|}
\hline & & 12 & 5 & \\
\hline & & 20 & 5 & \\
\hline & & 27 & 5 & \\
\hline & & 39 & 5 & \\
\hline RNA Seq & Species: & 5 & 4 & 12 \\
\hline Data set 2 & Nothobranchius & & & \\
\hline Kelmer et al., 2020 & furzeri & & & \\
\hline & Strain: MZM04/10 & & & \\
\hline & & 12 & 4 & \\
\hline & & 39 & 4 & \\
\hline Proteomic & Species: & 5 & 5 & 15 \\
\hline Data set & Nothobranchius & & & \\
\hline Kelmer et al., 2020 & furzeri & & & \\
\hline & Strain: MZM04/10 & & & \\
\hline & & 12 & 5 & \\
\hline & & 39 & 5 & \\
\hline & & 12 & 3 & \\
\hline & & 39 & 3 & \\
\hline Histology & Species: & 5 & 5 & 10 \\
\hline (EdU-ISH) & Nothobranchius & & & \\
\hline & furzeri & & & \\
\hline & Strain: MZM04/10 & & & \\
\hline & & 27 & 5 & \\
\hline & & & & 63 \\
\hline
\end{tabular}

2 min at $94^{\circ} \mathrm{C}$. In order to avoid drying out the slides, the whole process has been carried out in a wet chamber with PBS.

After hybridization, $2 \times$ saline-sodium citrate (SSC) has been used to remove the coverslip. Slides were first washed in $2 \times$ SSC, twice for $20 \mathrm{~min}$, and then in $0.2 \times$ SSC twice for $20 \mathrm{~min}$, always at hybridization temperature. A final washing step was done in PBT three times for $5 \mathrm{~min}$ at room temperature.

For the probe revelation, slides were incubated with blocking solution for $30 \mathrm{~min}$ at room temperature and then with Anti-DigAP Fab Fragments Ab [1/2,000] in blocking solution overnight at $4^{\circ} \mathrm{C}$.

Washing in PBT, three times for $5 \mathrm{~min}$, and in NMNT (NaCL/trisHCl/MgCl2/Tween20 0,1\%/ Tetramisole solution), three times for $5 \mathrm{~min}$ at room temperature, has been conducted before adding Fast Red solution (Roche Tablets; 1 in 2 ml Tris$\mathrm{HCl} 0.1 \mathrm{M}, \mathrm{pH}=8.2$ ). To avoid the formation of precipitate, Fast Red tablets have been vortexed for $5 \mathrm{~min}$ in Tris- $\mathrm{HCl}$ and then filtered. Observation has been conducted every 20 min with a Zeiss fluorescence microscope until the signal detection. The staining has been stopped by washing well with PBS (at least three times for $5 \mathrm{~min}$ ) at room temperature.

The young and old samples have been treated in parallel, and to be able compare the dimension of the expression domains between different ages, we stopped the colorimetric reaction in both at the same time. After ISH slides were treated for 
EdU immunolabeling, images were acquired using a confocal microscope (Leica TCS).

\section{EdU Staining}

After the ISH procedure, slides were processed to stain the population of proliferating cells in the neurogenic niches: $\mathrm{EdU}^{+}$ cells were fluorescently labeled with a bright photostable Alexa Fluor $^{\circledR} 488$ dye in a fast, highly specific click reaction, according to the purchaser's instruction.

Finally, slides were closed with a specific mounting (Fluoroshield, Sigma) and analyzed with a confocal microscope (Leica TCS).

\section{Cloning of Notch1a and Notch3 and Probe Preparation}

Total RNA was extracted from $N$. furzeri whole brains using miRNeasy Mini Kit (Qiagen), then total cDNA was retro transcribed from $1 \mu \mathrm{g}$ of RNA. PCR was performed on CDNA using a GoTaq polymerase (Promega), $60^{\circ} \mathrm{C}$ for annealing temperature, and $60 \mathrm{~s}$ elongation time using the following primers:

\section{Notch1aF: GCCCGACCATCCTTTTCTGA \\ NotchlaT7-R: GCAATGCAGAAGCCCTACTC-GGCCG GGACAAGTGCAATACC \\ Notch3F: AATGCTTGTCAAACCCGTGC \\ Notch3T7-R: CATCTGTCTGGATCCCTCGC-GGCCGG GACAAGTGCAATACC.}

Primers were designed using the NCBI Primer-BLAST, and the RNA sequences were identified on the NCBI website and were, respectively:

notch3 GenBank: HADY01014643.1

notch1a GenBank: HADY01022403.1.

$0.5 \mu \mathrm{g}$ of PCR products that contain T7 RNA polymerase promoter at the 3' ends were used as templates for in vitro transcription. The sequence of the amplicon was sent for direct sequencing (Genechrome, Rome, Italy) to confirm the identity of the amplified probe.

Probes were transcribed using DIG RNA labeling kit (SP6/T7) (Roche), according to the manufacturer's protocol.

\section{RNA Sequencing Data Analysis}

To assess gene expression of notch pathway and proliferation genes, we realized graphics of RNA expression starting from RNA-seq datasets derived from publicly available and published cross-sectional experiments (Baumgart et al., 2014 ${ }^{1}$; Kelmer et al., $2020^{2}$ ). We combined two RNA-seq datasets of brain aging in $N$. furzeri: a dataset covering five time points $(5,12,20,27$, and 39 weeks) and five biological replicates for each age and the second containing four replicates per age $(5,12$, and 39 weeks). These ages correspond to sexual maturity, young adult, adult (as defined by a decrease in growth rate), median life span, and old ( $\sim 30 \%$ survivorship) (Baumgart et al., 2014). In total, these represent 37 different samples spanning five different ages.

${ }^{1}$ http://www.ncbi.nlm.nih.gov/geo/query/acc.cgi?acc=GSE52462

${ }^{2}$ http://www.ncbi.nlm.nih.gov/geo/query/acc.cgi?acc=GSE125373
All the pieces of information concerning the age, strain, and number of animals considered for the analysis are summarized in Table 1. We first applied the function Deseq in the suite $\mathrm{R}$ to normalize the counts (to have more information about the normalization algorithm used by the Deseq 2 package, please refer to the paper by Love et al., 2014, and to the package manual at the link: http://www.bioconductor.org/packages/release/bioc/ vignettes/DESeq2/inst/doc/DESeq2.html). We then extracted the normalized counts of the two datasets and divided them for the average expression of their respective 5 week samples to be able to combine them.

We also analyzed a publicly available proteomic dataset (Kelmer et al., 2020 ${ }^{3}$ ). The dataset contains five replicates for age (5, 12, and 39 weeks, listed in Table 1) and is a combination of two separate experiments performed utilizing tandem mass tag and analyzing the same 12 week animals twice in two different contrasts: 39 vs. 12 weeks and 5 vs. 12 weeks. We therefore combined the data normalizing the protein expression dividing them for the mean value of the 12 weeks animals and plotted the resulting values. For both transcriptomics and proteomics data, we performed statistical analysis calculating the Spearman correlation value, the $p$-value adjusted, and the false discovery rate (FDR) to assess the statistical significance of the observed expression variability. All analyses were performed utilizing the suite R.

\section{RESULTS}

To obtain a global overview on the gene expression of members of the Notch family, we analyzed published RNA-seq (Baumgart et al., 2014), taking as the first time point of analysis the data obtained from 5-week-old animals, which corresponds to the time of sexual maturation followed by 12 weeks (young adult), 20 weeks (mature adult), 27 weeks (old), and 39 weeks (geriatric). Figure 1 reports the age-dependent regulation of gene expression coding for members of the Notch signaling pathway as identified by the Kyoto Encyclopedia of Genes and Genomes (KEGG) pathway (dre00430): eight Notch ligands, four receptors, and three effectors. Out of these, four ligands (dla, dlb, dld, and jag2), notch1a, and proliferative genes (mki67 and pcna) showed a negative correlation with age, indicating an age-dependent downregulation of the Notch pathway, and it is consistent with an age-dependent reduction of adult neurogenesis detected previously by neuroanatomical techniques (Tozzini et al., 2012) and RNA-seq (Baumgart et al., 2016). More precisely, the observed decrease is greater in the time frame between 5 and 12 weeks of age (Figure 1), while the downregulation decelerates between 12 and 27 weeks. Statistical significance of expression changes can be assessed by the correlation and $\boldsymbol{p}$-value data listed in Table 2.

The amplitude of regulation was largest for the delta ligands (with the exception of $d l c$ that, however, is $\sim 20$ times lower expressed than the other three delta genes) and notch1a. Although notch 3 expression shows the same downregulation

${ }^{3}$ http://www.ebi.ac.uk/pride/archive/projects/PXD012314 


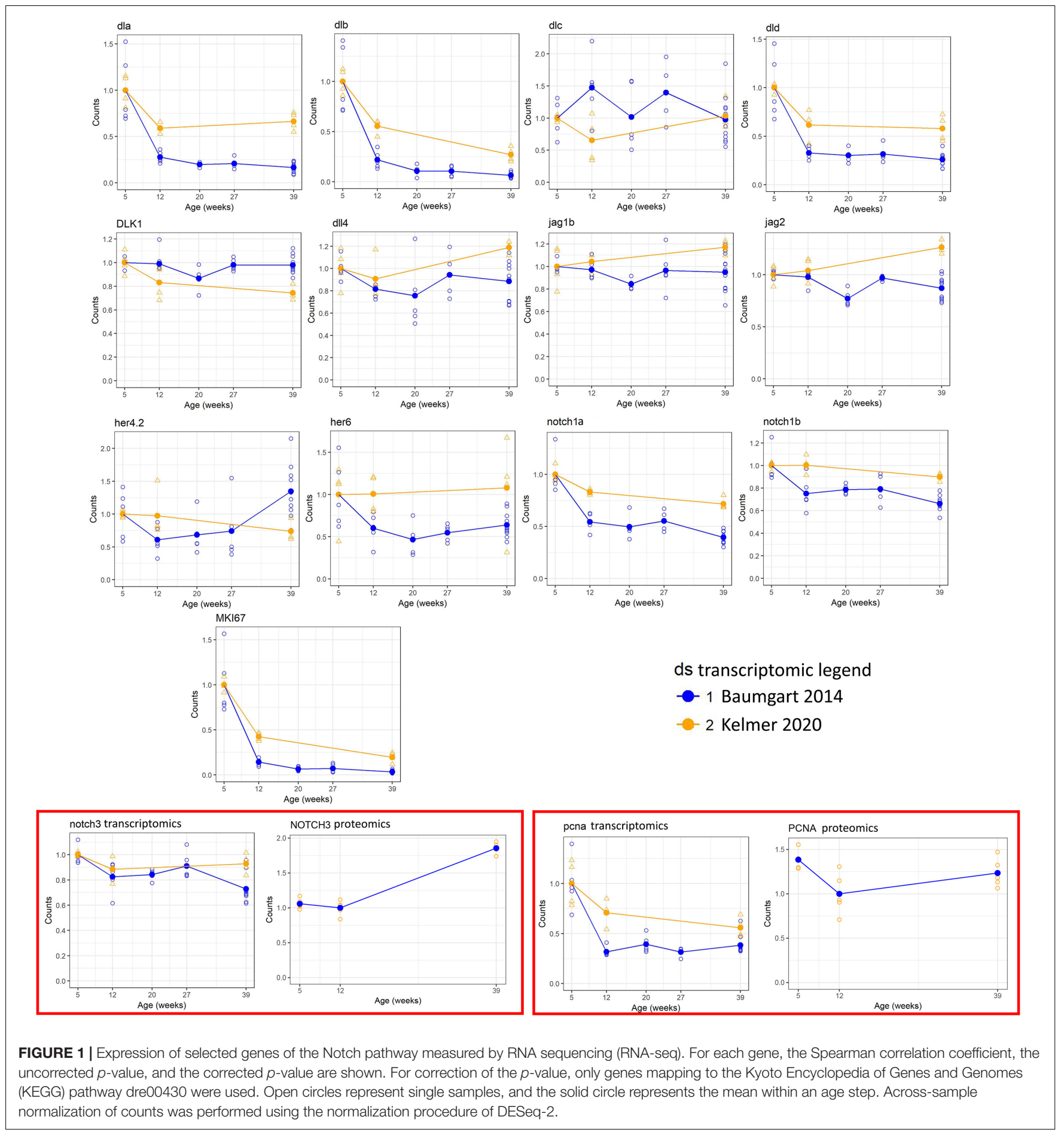

trend between 5 and 12 weeks, the overall reduction is consistently smaller, how attested by the relative RNA-seq data analysis (Figure 1) and the expression pattern in the brain tissue (Figure 2).

We were also able to find proteomic data regarding the levels of Notch3 and proliferating cell nuclear antigen (PCNA); even if the transcripts of the two genes show no evident age-dependent reduction, at the protein level, a mechanism of compensation seems to occur (Figure 1). A partial decoupling between RNA and protein level during aging already has been demonstrated in this model (Kelmer et al., 2020), and this observation could be included in this context, but from the data obtained, a Notch3 reduction can be excluded.

We decided to investigate the expression of notch1a (Gene ID Nfu_g_1_006683, annotated using the zebrafish ortholog ENSDARG00000052094) and notch3 (Gene ID Nfu_g_1_007618, 
TABLE 2 | Spearman correlation value and respective $p$-values and adjusted $p$-values for gene and proteins listed in Figure 1.

\begin{tabular}{|c|c|c|c|c|c|c|c|c|c|}
\hline \multirow[b]{2}{*}{ gene } & \multicolumn{3}{|c|}{ Transcriptomics, Dataset 1 Baumgart et al., 2014} & \multicolumn{3}{|c|}{ Transcriptomics, Dataset 2 Kelmer et al., 2020} & \multicolumn{3}{|c|}{ Proteomics Kelmer et al., 2020} \\
\hline & Spearman CV & pVal & FDR & Spearman CV & pVal & FDR & Spearman CV & pVal & FDR \\
\hline dla & $-7.54629 E+14$ & $\begin{array}{c}9.382986565 \\
49819 e-07\end{array}$ & $\begin{array}{l}6.00511140 \\
191884 \mathrm{e}-06\end{array}$ & $-5.91312 \mathrm{E}+14$ & $\begin{array}{c}0.0428679750 \\
394139\end{array}$ & $\begin{array}{c}0.07620973340 \\
34024\end{array}$ & & & \\
\hline$d l b$ & $-8.17445 E+14$ & $\begin{array}{c}1.996229181 \\
9553 \mathrm{e}-08\end{array}$ & $\begin{array}{l}3.19396669 \\
112848 \mathrm{e}-07\end{array}$ & $-9.461 E+14$ & $\begin{array}{c}3.271765152 \\
57932 \mathrm{e}-06\end{array}$ & $\begin{array}{l}2.093929697 \\
65076 \mathrm{e}-05\end{array}$ & & & \\
\hline$d l c$ & $-1.41441 \mathrm{E}+13$ & $\begin{array}{c}0.447867241 \\
867479\end{array}$ & $\begin{array}{c}0.5971563224 \\
89972\end{array}$ & $-2.95656 \mathrm{E}+14$ & $\begin{array}{c}0.9273255929 \\
17055\end{array}$ & $\begin{array}{c}0.9572393217 \\
20831\end{array}$ & & & \\
\hline dld & $-6.64356 \mathrm{E}+14$ & $\begin{array}{l}4.587876016 \\
78168 \mathrm{e}-05\end{array}$ & $\begin{array}{c}0.0001835150406 \\
71267\end{array}$ & $-7.39141 E+14$ & $\begin{array}{c}0.00601894061 \\
957786\end{array}$ & $\begin{array}{c}0.0128404066 \\
550994\end{array}$ & & & \\
\hline DLK1 & $-4.74243 E+14$ & $\begin{array}{c}0.800006284 \\
621884\end{array}$ & $\begin{array}{c}0.8258129389 \\
64525\end{array}$ & $-7.39141 E+14$ & $\begin{array}{c}0.00601894061 \\
957786\end{array}$ & $\begin{array}{c}0.01284040665 \\
50994\end{array}$ & & & \\
\hline dll4 & $-5.49124 \mathrm{E}+14$ & $\begin{array}{c}0.769219214 \\
254042\end{array}$ & $\begin{array}{c}0.8205004952 \\
04311\end{array}$ & $5.32181 E+14$ & $\begin{array}{c}0.07489 \\
8436\end{array}$ & $\begin{array}{c}0.1141309493 \\
5522\end{array}$ & & & \\
\hline her4.2 & 4.16003E + 14 & $\begin{array}{c}0.019930908 \\
1685124\end{array}$ & $\begin{array}{c}0.0455563615 \\
280283\end{array}$ & $-7.39141 E+14$ & $\begin{array}{c}0.006018940619 \\
57786\end{array}$ & $\begin{array}{c}0.01284040665 \\
50994\end{array}$ & & & \\
\hline her6 & $-2.25889 E+13$ & $\begin{array}{l}0.221751400 \\
583059\end{array}$ & $\begin{array}{c}0.4174144010 \\
97524\end{array}$ & $5.91312 E+14$ & $\begin{array}{c}0.8551579930 \\
78424\end{array}$ & $\begin{array}{c}0.9121685259 \\
50319\end{array}$ & & & \\
\hline jag1b & $-5.65764 \mathrm{E}+14$ & $\begin{array}{c}0.7624224206 \\
48025\end{array}$ & $\begin{array}{c}0.8205004952 \\
04311\end{array}$ & $4.7305 E+14$ & $\begin{array}{c}0.1203734281 \\
97896\end{array}$ & $\begin{array}{c}0.1750886228 \\
33304\end{array}$ & & & \\
\hline jag2 & $-4.05603 E+14$ & $\begin{array}{c}0.02358690140 \\
07746\end{array}$ & $\begin{array}{c}0.05031872298 \\
83191\end{array}$ & $7.68706 \mathrm{E}+14$ & $\begin{array}{c}0.0034824680 \\
3771213\end{array}$ & $\begin{array}{c}0.00928658143 \\
389901\end{array}$ & & & \\
\hline MKI67 & $-8.55302 E+14$ & $\begin{array}{c}8.8838891798 \\
5594 \mathrm{e}-10\end{array}$ & $\begin{array}{c}2.84284453755 \\
39 \mathrm{e}-08\end{array}$ & $-9.461 E+14$ & $\begin{array}{c}3.271765152 \\
57932 \mathrm{e}-06\end{array}$ & $\begin{array}{c}2.0939296976 \\
5076 \mathrm{e}-05\end{array}$ & & & \\
\hline notch1a & $-6.48132 E+14$ & $\begin{array}{c}8.064381403 \\
0168 \mathrm{e}-05\end{array}$ & $\begin{array}{c}0.000258060204 \\
896537\end{array}$ & $-6.80009 E+14$ & $\begin{array}{c}0.0149674939 \\
835605\end{array}$ & $\begin{array}{c}0.0299349879 \\
671209\end{array}$ & & & \\
\hline notch1b & $-7.75429 E+14$ & $\begin{array}{c}3.0068523781 \\
9565 \mathrm{e}-07\end{array}$ & $\begin{array}{c}3.20730920340 \\
869 \mathrm{e}-06\end{array}$ & $-9.461 E+14$ & $\begin{array}{c}3.2717651525 \\
7932 \mathrm{e}-06\end{array}$ & $\begin{array}{c}2.0939296976 \\
5076 e-05\end{array}$ & & & \\
\hline notch3 & $-5.92804 \mathrm{E}+13$ & $\begin{array}{c}0.000441182018 \\
497299\end{array}$ & $\begin{array}{c}0.00117648538 \\
265946\end{array}$ & $-4.13919 E+14$ & $\begin{array}{c}0.181014993 \\
978156\end{array}$ & $\begin{array}{c}0.251846948 \\
143521\end{array}$ & $\begin{array}{c}0.64253962 \\
0399475\end{array}$ & $\begin{array}{c}0.009787763993 \\
86838\end{array}$ & $\begin{array}{c}0.0261977326 \\
229934\end{array}$ \\
\hline pcna & $-2.04257 E+14$ & $\begin{array}{c}0.2703778006 \\
73789\end{array}$ & $\begin{array}{c}0.4806716456 \\
42292\end{array}$ & $-8.27837 E+14$ & $\begin{array}{c}0.00088528528 \\
9653703\end{array}$ & $\begin{array}{c}0.0025753753 \\
880835\end{array}$ & $\begin{array}{c}-0.3023715913 \\
29575\end{array}$ & $\begin{array}{c}0.2733497681 \\
76361\end{array}$ & $\begin{array}{c}0.3629205847 \\
07689\end{array}$ \\
\hline
\end{tabular}

FDR, false discovery rate. 


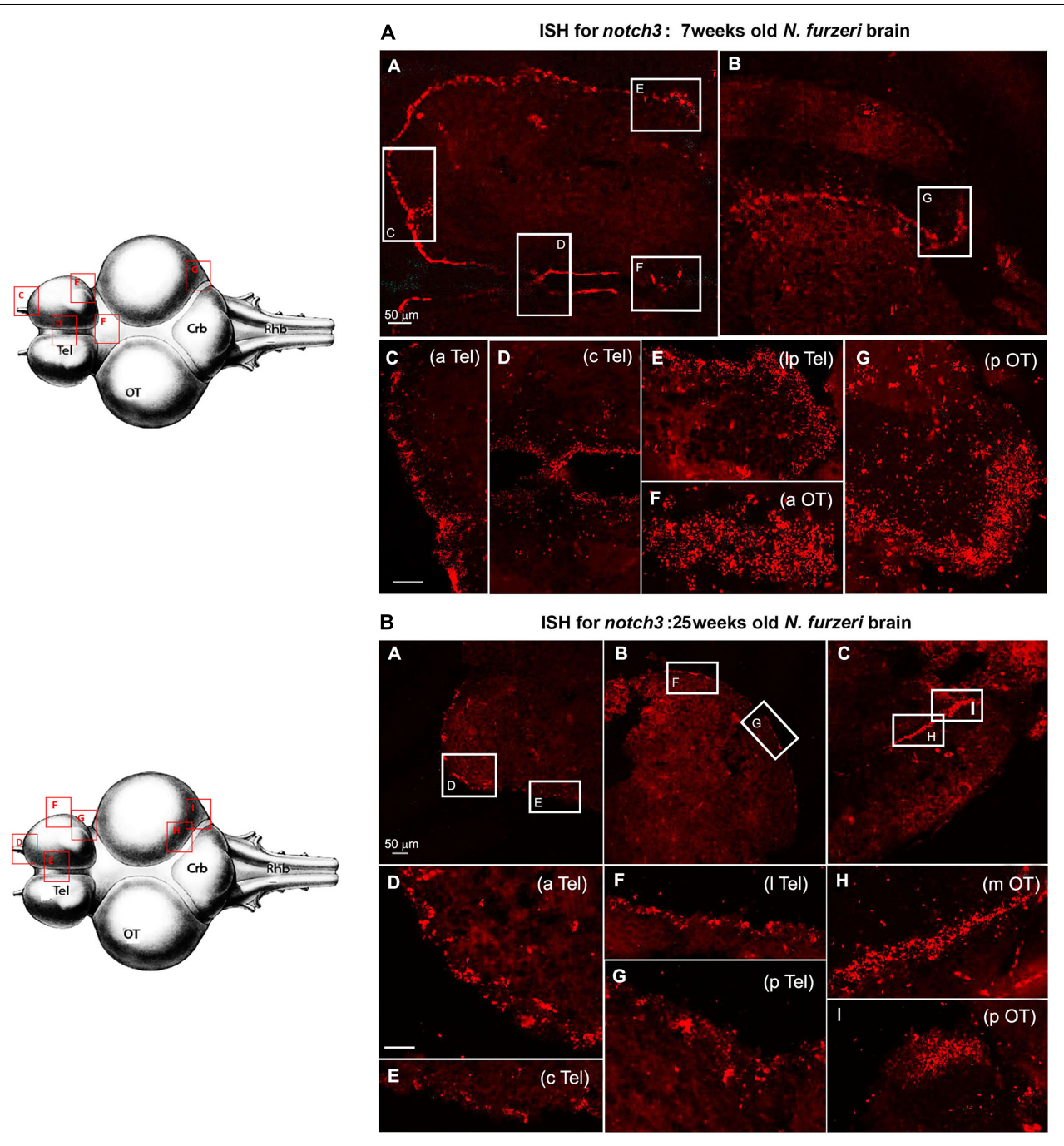

FIGURE 2 | Notch3 expression in proliferative niches of 7-weeks-old (A: A-G) vs. 25-weeks-old (B: A-F) N. furzeri brain. (A) (A,B) Overview of the brain in horizontal medial section (along the dorso/ventral axis) stained for notch1a, respectively, in telencephalon (A) and optic tectum (OT) (B) regions of a young N. furzeri brain (7 weeks). White frames show the areas magnified in panels (C-G), representing, respectively, the anterior (aTel), central (cTel), and lateroposterior (IpTel) telencephalic regions and the anterior (aOT) and posterior optic tectum regions. (B) (A-C) Overview of the brain in horizontal medial section (along the dorso/ventral axis) stained for notch3, respectively, in the anterior (A) and lateroposterior (B) telencephalon and the posterior optic tectum (pOT) (C) regions of an old N. furzeri brain (25 weeks). White frames show the areas magnified in panels (D-I), representing, respectively, the anterior (aTel), central (cTel), lateral (ITel), and posterior (pTel) telencephalic regions and the anterior (aOT) and posterior (pOT) optic tectum regions. Scale bars always represent $50 \mu \mathrm{m}$ and are indicative for all further images without specific scale bar indication. Brain drawings on the left (modified from D'angelo, 2013-Atlas) represent the horizontal overview of the whole killifish brain; red-squared areas indicate the position of the figure panels based on the corresponding letters.

annotated using the zebrafish ortholog ENSDARG00000052139). As described in the Introduction, these genes play different roles in controlling NSC proliferation and differentiation in the zebrafish brain (Alunni et al., 2013; de Oliveira-Carlos et al., 2013). Although both genes are downregulated with age, the amplitude of regulation (i.e., 5 vs. 39 weeks) is different: $40 \%$ for notch $1 a$ vs. $15 \%$ for notch 3 .

In order to localize the expression domains of notch1a and notch 3 in the $N$. furzeri brain, we performed ISH on horizontal sections concentrating on the neurogenic niches of the telencephalon and optic tectum that we previously described in detail (Tozzini et al., 2012).
Figure 3 (modified from Tozzini et al., 2012) shows a wholemount brain of young $N$. furzeri, where all neurogenic niches are visualized by incorporation of EdU and green fluorescent labeling ( $4 \mathrm{~h}$ after injection) and serves as a reference for the ensuing ISH images of the study.

The messenger of notch1a was coexpressed, although not exclusively limited, to active neurogenic niches, as demonstrated by double labeling with the mitotic marker EdU (Figure 4). In the telencephalon, expression of notch1a was mostly seen in $\mathrm{EdU}^{-}$cells that were close neighbors of a notch1a ${ }^{-} \mathrm{EdU}^{+}$ cell, as expected, given the antimitotic activity of Notch pathway activation. A few cells were double labeled for notch $1 a$ and 


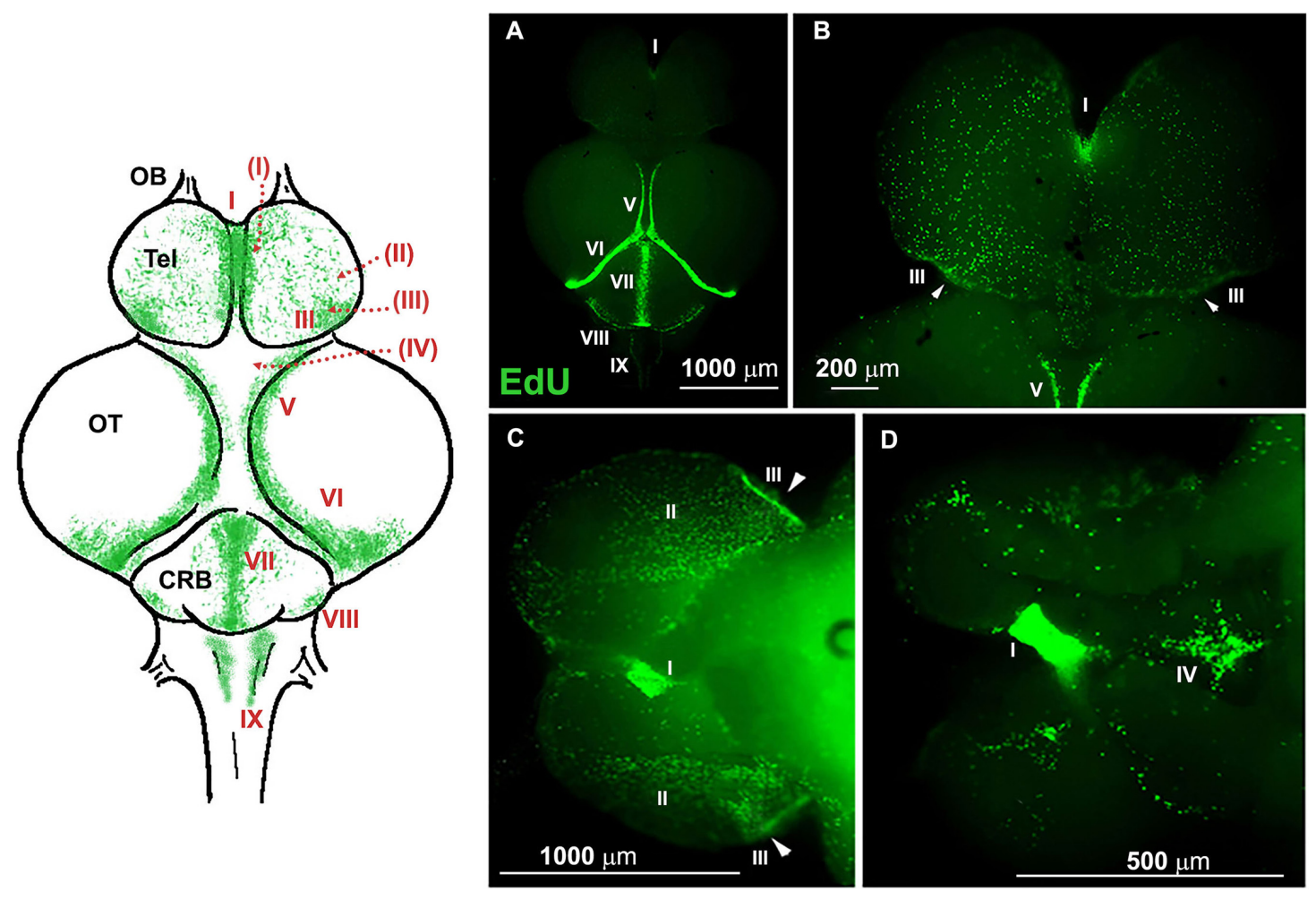

FIGURE 3 | Whole-mount (WM) overview of ethynyl deoxyuridine (EdU)+ cells in 7-weeks-old Nothobranchius furzeri, $4 \mathrm{~h}$ after intraperitoneal injection (modified from Tozzini et al., 2012). A schematic drawing of the dorsal view of $N$. furzeri brain is shown on the left, where all principal brain regions are defined (OB, olfactory bulb; Tel, telencephalon; OT, optic tectum; CRB, cerebellum), and neurogenic niche distribution indicated by green dots and sequential Roman numeral indexes; dotted red lines indicate the extension of a niche (or part of it) on the ventral side of the cerebral structure, here not represented. (A) Dorsal view of the brain.

(B) Telencephalic region. (C,D) Ventral view of the brain at lower (C) and higher (D) magnification. Nine neurogenic niches are identified in these panels: I, telencephalic niche corresponding to the subpallial region; II and III, telencephalic niches corresponding to the pallial regions; IV, preoptic niche; V, rostro-dorsal part of the niche in the optic tectum (OT); VI, caudal part of niche in the OT; VII and VIII, medial and caudal niches of the cerebellum; IX, caudal niche along the roof of the IV ventricle, visible in panel (A). Arrowheads (B,D) indicate areas of higher concentration of proliferating cells in the caudal margin of niche III.

EdU (white arrows in Figures $\mathbf{4 F , G}$ ), as already reported in zebrafish (Alunni et al., 2013). It should be noticed that notch1a expression in the telencephalon was more prominent in the anterior and medial parts of the telencephalon (Figures 4EG) that correspond to a subpallial highly mitotic area (area I in Figure 3), while the number of labeled cells is smaller in the lateral margin of the telencephalon that is a pallial, low active mitotic area (area II in Figure 3). Expression becomes higher in a lateroposterior area where mitotic activity is moderate (Figure 4B, area III in Figure 3). In the optic tectum, expression of notch $1 a$ is strictly limited to neuroepithelial stem cell niches (Figures 4C,D) where partial double labeling of notch1a and EdU is observed.

In agreement with the RNA-seq data (Figure 1), expression of notch $1 a$ markedly declines during aging in all neurogenic niches without changing the regional pattern of labeling (Figure 5).

Although the RNA-seq data show a minor drop in the notch3 expression between 5 and 12 weeks of age, our ISH results suggest that already at 7 weeks, we can observe a level of notch3 RNA expression that is comparable to the level observed at 27 weeks of age. As opposed to notch1a, the expression of notch 3 was not limited to active neurogenic niches (EdU positive), but it was equally observed in all stem cell niches, regardless of the visible mitotic activity level in the region (Figure 5). In the young adult telencephalon, expression of notch 3 was similar in regions that vary greatly in mitotic level: the subpallial highly mitotic area (Figures 6A-D, area I in Figure 3) throughout the frontal margin of the telencephalon, that is, a pallial, lower active mitotic area (Figures 6A-C), and in the lateroposterior area with moderate mitotic activity (Figures 6A-E, area II and III in Figure 3). In the optic tectum, expression of notch3 is not limited to neuroepithelial stem cell niches (Figures 6F-G, anterior and posterior margin niches of the OT, respectively), but extends into the ventricular margin of the optic tectum (Figure 6B) which clearly shows only a red staining and it is formed by radial glia with extremely low mitotic activity (Tozzini et al., 2012).

\section{DISCUSSION}

In conclusion, by comparing the RNA-seq results with what has emerged from the histological analysis of the ISH experiments, both expression pattern and regulation differ between notch 1a and notch3. Expression of the former is limited to mitotically 

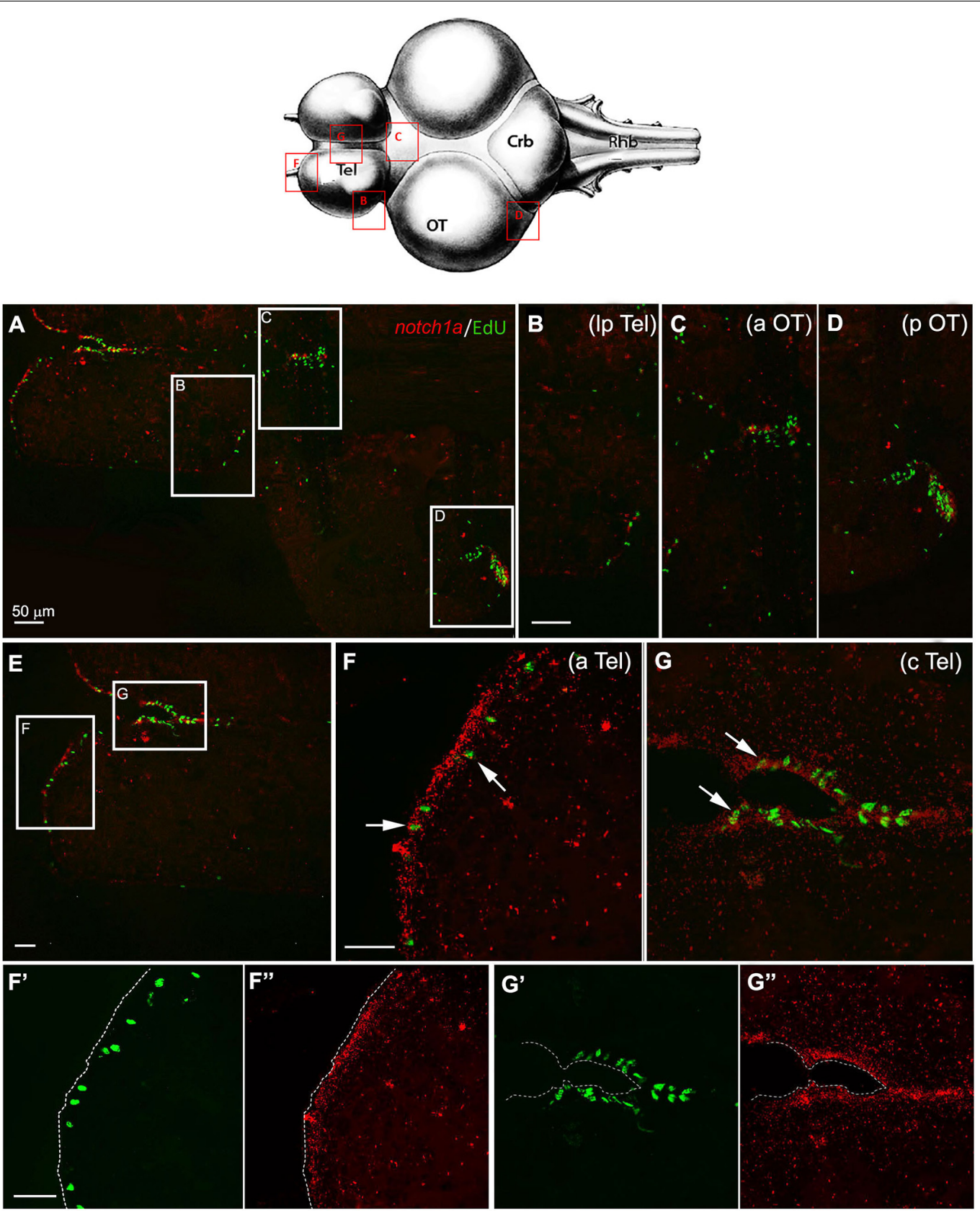

FIGURE 4 | Notch1a expression in proliferative niches of 7-weeks-old N. furzeri brain. (A) Overview of the brain in horizontal medial section (along the dorso/ventral axis) double-stained for ethynyl deoxyuridine (EdU) in green (labeling S-phase cells) and notch1a in red [by in situ hybridization (ISH)]. White frames show the areas magnified in panels (B-D), representing, respectively, the lateroposterior telencephalon (IpTel), the anterior (aOT) and posterior optic tectum (E) representation of the telencephalic structure, labeled for EdU and notch1. White frames show the areas magnified in panels (F,G), representing, respectively, the anterior (aTel) and central

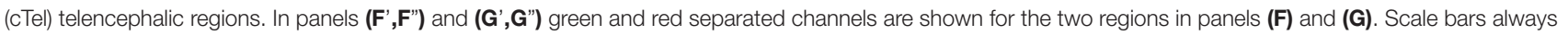
represent $50 \mu \mathrm{m}$ and are indicative for all further images without specific scale bar indication. The upper brain drawing (modified from D'angelo, 2013-Atlas) represents the horizontal overview of the whole killifish brain; red-squared areas indicate the position of the figure panels based on the corresponding letters. White arrows show the presence of double-stained Edu+/notch1a cells.

active regions and consequently is greatly reduced by aging. Expression of the latter is observed in all cells that have a neurogenic potential, regardless of the level of their actual mitotic activity, and is less influenced by age both at the transcript and protein levels.
The reduction of the expression of notch1a (as well as that of mki67 and pcna) shows the bigger amplitude between the ages of 5 and 12 weeks, the period in which the brain grows considerably. Based on the techniques utilized in this work (RNA-seq data analysis and ISH), it 


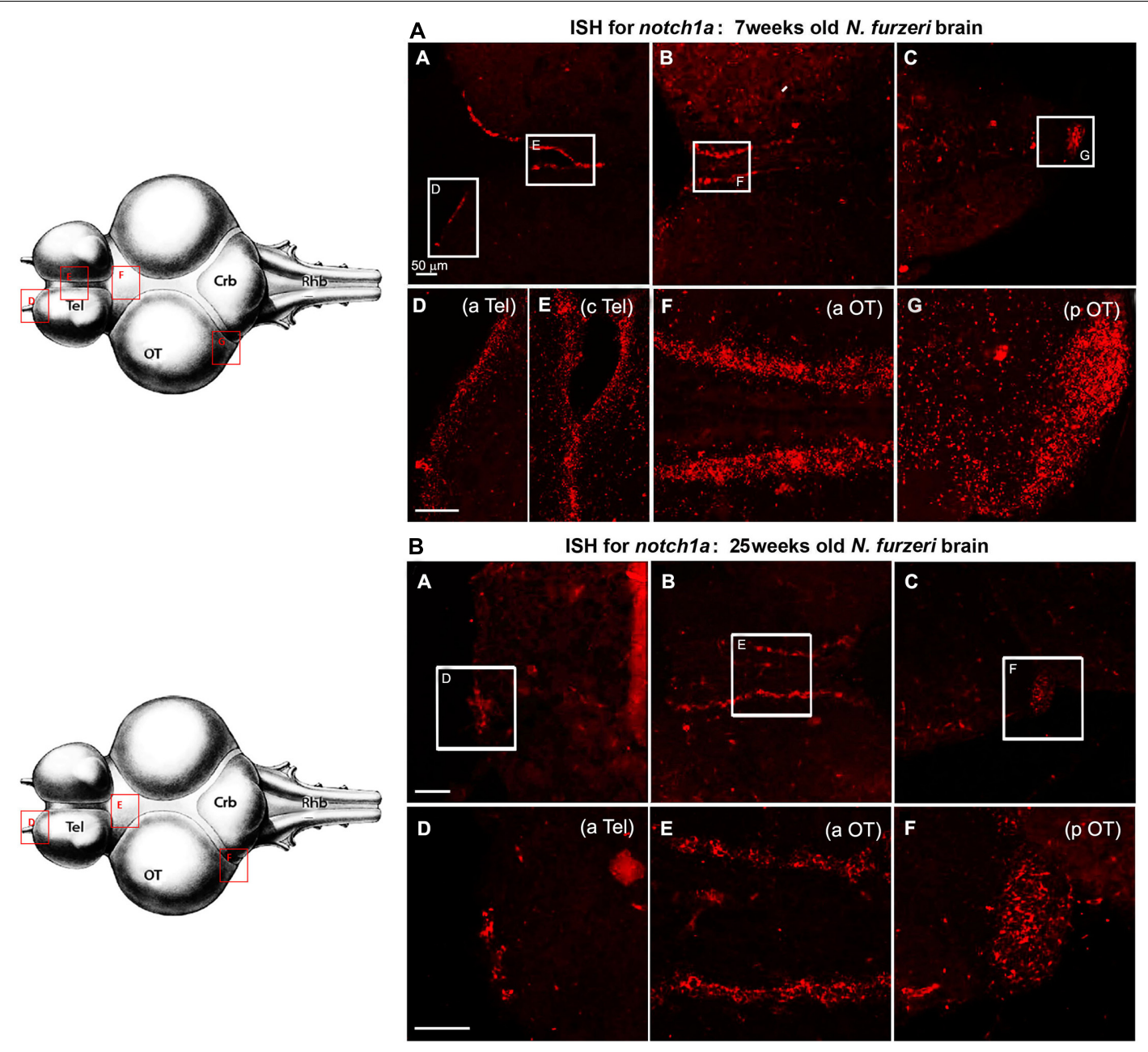

FIGURE $\mathbf{5}$ | Notch1 expression in proliferative niches of 7-weeks-old (A: A-G) vs. 25-weeks-old (B: A-F) N. furzeri brain. (A) (A-C) Overview of the brain in horizontal medial section (along the dorso/ventral axis) stained for notch1a, respectively, in telencephalon (A), anterior optic tectum (aOT) (B), and posterior optic tectum (pOT) (C) regions of a young N. furzeri brain (7 weeks). White frames show the areas magnified in panels (D-G), representing, respectively, the anterior (aTel) and central (cTel) telencephalic regions and the anterior (aOT) and posterior optic tectum regions. (B) (A-C) Overview of the brain in horizontal medial section (along the dorso/ventral axis) stained for notch1a, respectively, in telencephalon (A), anterior optic tectum (aOT) (B) and posterior optic tectum (pOT) (C) regions of an old N. furzeri brain (25 weeks). White frames show the areas magnified in panels (D-G), representing, respectively, the anterior (aTel) and central (cTel) telencephalic regions and the anterior (aOT) and posterior (pOT) optic tectum regions. Scale bars always represent $50 \mu \mathrm{m}$ and are indicative for all further images without specific scale bar indication. Brain drawing on the left (modified from D'angelo, 2013-Atlas) represents the horizontal overview of the whole killifish brain; red-squared areas indicate the position of the figure panels based on the corresponding letters.

cannot be formally excluded that the reduction observed could be due to the relative reduction in the fraction of cells expressing notch1a on the total amount of cells in the brain. However, the fact that its normalized expression is reduced at the brain level is nonetheless a significant observation. Typically, all pathways involved in growth and differentiation are strongly involved in the metabolic processes that determine the future aging and life span of an individual; it also has been demonstrated that the specific expression of metabolic-related genes already at early life stages could be predictive of life expectancy of Nothobranchius (Baumgart et al., 2016). Thus, growth and aging are two processes that are strictly correlated and cannot be easily disentangled.
On the other hand, notch 3 transcript seems to be less affected by aging and even to increase its protein level over time. Unfortunately, the dataset that we analyzed did not contain information about Notchla or other Notch proteins.

From the literature, it is known that the reduction of notch1a induces stem cells to divide asymmetrically, whereas the reduction of notch 3 promotes symmetrical divisions (Alunni et al., 2013; Than-Trong et al., 2018). Thus, a different antiproliferative role could be inferred for the two genes, depending on the differential proliferation outcome.

Our results show that in $N$. furzeri telencephalon, notch1a is expressed, although not exclusively, in proliferating regions, mainly localizing in close proximity to $\mathrm{EdU}^{+}$cells, areas in which the expression of notch 1 a seems to be more concentrated. 

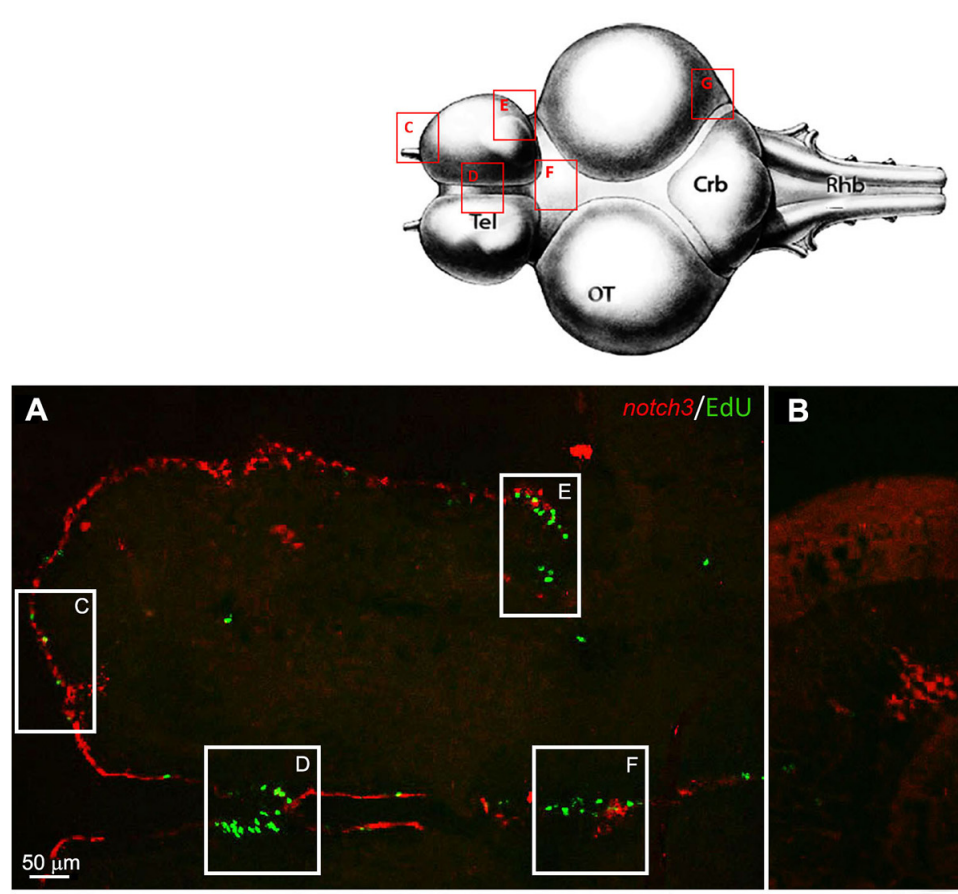

B

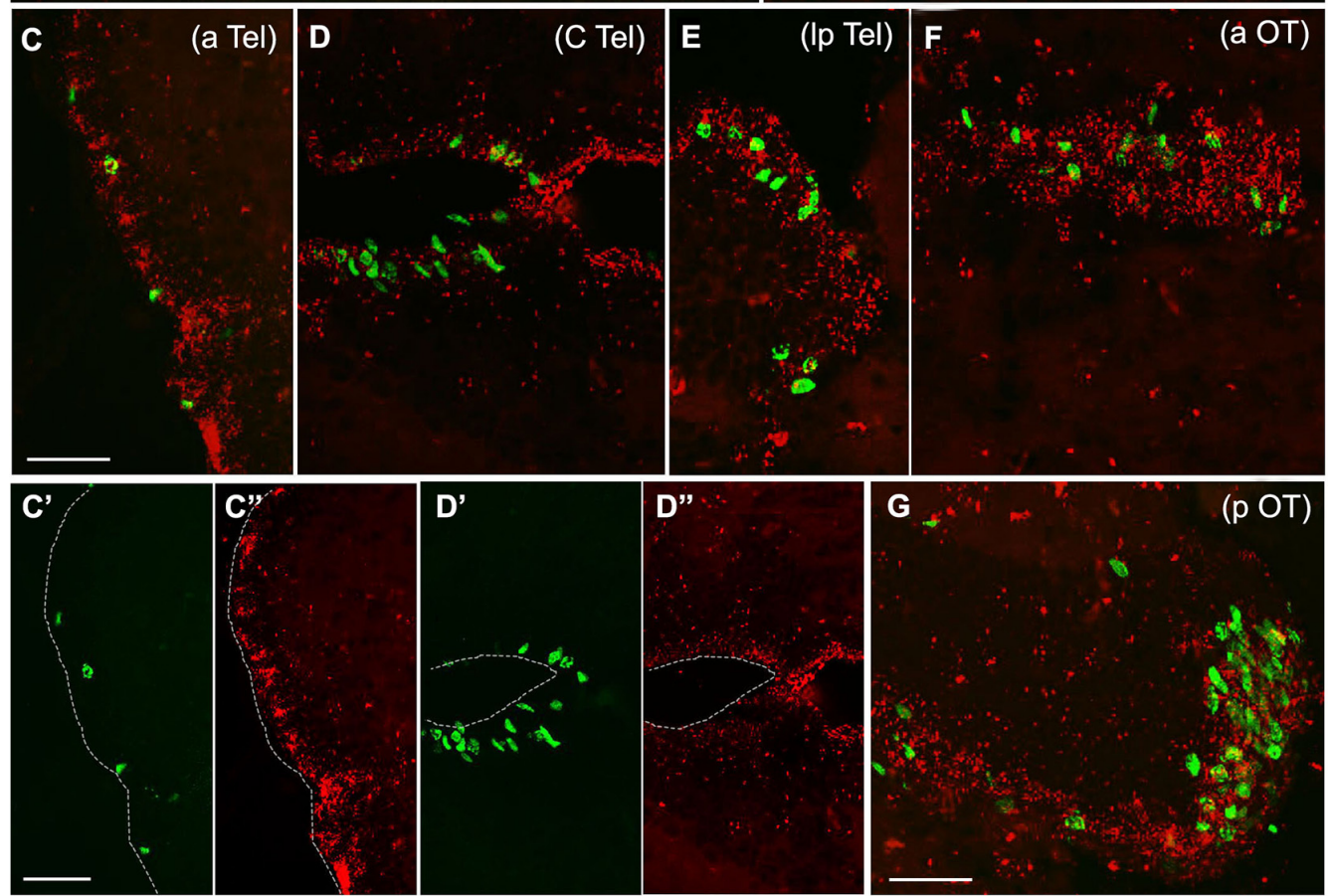

FIGURE 6 | Notch3 expression in proliferative niches of 7 weeks-old N. furzeri brain: (A) Overview of the brain in horizontal medial section (along the dorso/ventral axis) double-stained for EdU in green (labelling S-phase cells) and notch3 in red (by ISH). White frames show the areas magnified in (C-G), representing respectively

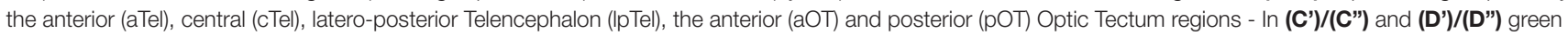
and red separated channel are shown for the two regions in (C,D) - Scale bars always represent 50 $\mathrm{m}$ and are indicative for all further images without specific scale bar indication. Footprint (modified from D'angelo, 2013-Atlas) represents the horizontal overview of the whole killifish brain; red-squared areas indicate the position of the figure panels, based on the corresponding letters. White arrows show the presence of double-stained Edu $+/$ notch3 cells.

In zebrafish, it has been described that the high majority of proliferative radial glia and about $50 \%$ of proliferative non-radial glia express notch1a (de Oliveira-Carlos et al., 2013), while in killifish, we observed that almost all proliferative $\mathrm{Edu}^{+}$cells of the telencephalic niches do not express notch1a, supporting the notion of the antiproliferative role of this gene, as also 
supported by the work of Chapouton et al. (2011), in which they showed the mutually exclusive expression of proliferative markers and effectors of Notch pathway. The limited number of double-stained EdU-notch1a cells (Figure 4 white arrows) could represent cells lacking the expression of notch3, thus undergoing the symmetrical above the asymmetrical division (inhibited by notch $1 a$ expression). These cells could belong to the pool of slowcycling, glial-like NSCs, already observed in our previous work where double-stained $\mathrm{S}_{100}{ }^{+} / \mathrm{EdU}^{+}$cells were present in this area in exiguous number (Tozzini et al., 2012).

In zebrafish telencephalon, notch3 has been described to have an expression pattern similar to notch1a in radial glial cells but to be rarely expressed in the non-glial proliferative population (de Oliveira-Carlos et al., 2013). In our observations, notch3 shows a more widespread area of staining with respect to the neurogenic niches, but also in this case, we could identify a few double-labeled $\mathrm{EdU}^{+} /$notch ${ }^{+}$cells.

In zebrafish optic tectum, the majority of proliferating cells are reported to express notch1a and notch3 (de OliveiraCarlos et al., 2013). In our case, notch1a expression resembles closely the situation already described for the telencephalon: very few cells, both in the anterior and posterior neurogenic niches, present a double Edu/notchla staining. On the contrary, notch 3 seems to have a higher level of colocalization with $\mathrm{EdU}^{+}$cells with respect to notch1a. The interpretation of this finding is ambiguous: it is known that neurogenic niches of the optic tectum have in fish a neuroepithelial origin (Ito et al., 2010) and are characterized by fast-cycling cells supposedly undergoing symmetrical divisions. It is possible that in these areas, notch 3 does not have the canonical function of symmetrical division inhibition. To assess that, further investigations would be needed.

Taken together, our observations finally suggest that, as already demonstrated for zebrafish (Alunni et al., 2013), also in our model, a possible differential role of the two receptors could exist in neurogenic activity control by acting on distinct cellular

\section{REFERENCES}

Adolf, B., Chapouton, P., Lam, C. S., Topp, S., Tannhauser, B., Strahle, U., et al. (2006). Conserved and acquired features of adult neurogenesis in the zebrafish telencephalon. Dev. Biol. 295, 278-293. doi: 10.1016/j.ydbio.2006.03. 023

Allard, J. B., Kamei, H., and Duan, C. (2013). Inducible transgenic expression in the short-lived fish Nothobranchius furzeri. J. Fish Biol. 82, 1733-1738. doi: $10.1111 /$ jfb.12099

Alunni, A., Krecsmarik, M., Bosco, A., Galant, S., Pan, L., Moens, C. B., et al. (2013). Notch3 signaling gates cell cycle entry and limits neural stem cell amplification in the adult pallium. Development 140, 3335-3347. doi: 10.1242/dev.095018

Baumgart, M., Groth, M., Priebe, S., Savino, A., Testa, G., Dix, A., et al. (2014). RNA-seq of the aging brain in the short-lived fish N. furzeri - conserved pathways and novel genes associated with neurogenesis. Aging Cell 13, 965-974. doi: 10.1111/acel.12257

Baumgart, M., Priebe, S., Groth, M., Hartmann, N., Menzel, U., Pandolfini, L., et al. (2016). Longitudinal RNA-Seq analysis of vertebrate aging identifies mitochondrial complex I as a small-molecule-sensitive modifier of lifespan. Cell Syst. 2, 122-132. doi: 10.1016/j.cels.2016.01.014

Ben Abdallah, N. M., Slomianka, L., Vyssotski, A. L., and Lipp, H. P. (2010). Early age-related changes in adult hippocampal neurogenesis in C57 mice. Neurobiol. Aging 31, 151-161. doi: 10.1016/j.neurobiolaging.2008.03.002 subpopulations, so to realize a fine-tuned regulation of the proliferative potential of the niches throughout the entire fish life.

\section{DATA AVAILABILITY STATEMENT}

Publicly available datasets were analyzed in this study. This data can be found here: Baumgart et al. (2014): http://www. ncbi.nlm.nih.gov/geo/query/acc.cgi?acc=GSE52462; Kelmer et al. (2020): http://www.ncbi.nlm.nih.gov/geo/query/acc.cgi?acc= GSE125373; Kelmer et al. (2020): http://www.ebi.ac.uk/pride/ archive/projects/PXD012314.

\section{ETHICS STATEMENT}

The animal study was reviewed and approved by Italian Ministry of Health (Number 96/2003a).

\section{AUTHOR CONTRIBUTIONS}

ETT designed the study. ETT and SB performed in situ hybridization, confocal acquisition, imaging, and wrote the manuscript. SB performed RNAseq analysis. Both authors read and approved the final manuscript.

\section{ACKNOWLEDGMENTS}

We thank Alessandro Cellerino for providing logistic and scientific support, Giorgia Battistoni for helping to perform the in situ hybridization experiments and confocal acquisitions, and Roberto Ripa for helping to perform the cloning of the probes. This work was partially supported by the MFAG 11511 (AIRC Association) to ETT.

Benayoun, B. A., Pollina, E. A., and Brunet, A. (2015). Epigenetic regulation of ageing: linking environmental inputs to genomic stability. Nat. Rev. Mol. Cell. Biol. 16, 593-610. doi: 10.1038/nrm4048

Bierkamp, C., and Campos-Ortega, J. A. (1993). A zebrafish homologue of the Drosophila neurogenic gene Notch and its pattern of transcription during early embryogenesis. Mech. Dev. 43, 87-100. doi: 10.1016/0925-4773(93)90027-u

Blazek, R., Polacik, M. J., and Reichard, M. (2013). Rapid growth, early maturation and short generation time in African annual fishes. Evodevo 4:24.

Cellerino, A., Valenzano, D. R., and Reichard, M. (2016). From the bush to the bench: the annual Nothobranchius fishes as a new model system in biology. Biol. Rev. Camb. Philos. Soc. 91, 511-533. doi: 10.1111/brv.12183

Chapouton, P., Webb, K. J., Stigloher, C., Alunni, A., Adolf, B., Hesl, B., et al. (2011). Expression of hairy/enhancer of split genes in neural progenitors and neurogenesis domains of the adult zebrafish brain. J. Comp. Neurol. 519, 1748-1769. doi: 10.1002/cne.22599

Cui, R., Willemsen, D., and Valenzano, D. R. (2020). Nothobranchius furzeri (African Turquoise Killifish). Trends Genet. 36, 540-541. doi: 10.1016/j.tig.2020. 01.012

D'angelo, L. (2013). Brain atlas of an emerging teleostean model: nothobranchius furzeri. Anat. Rec. 296, 681-691. doi: 10.1002/ar.22668

de Oliveira-Carlos, V., Ganz, J., Hans, S., Kaslin, J., and Brand, M. (2013). Notch receptor expression in neurogenic regions of the adult zebrafish brain. PLoS One 8:e73384. doi: 10.1371/journal.pone.0073384 
Di Cicco, E., Tozzini, E. T., Rossi, G., and Cellerino, A. (2011). The shortlived annual fish Nothobranchius furzeri shows a typical teleost aging process reinforced by high incidence of age-dependent neoplasias. Exp. Gerontol. 46, 249-256. doi: 10.1016/j.exger.2010.10.011

Dolfi, L., Ripa, R., Antebi, A., Valenzano, D. R., and Cellerino, A. (2019). Cell cycle dynamics during diapause entry and exit in an annual killifish revealed by FUCCI technology. Evodevo. 10:29. doi: 10.1186/s13227-019-0142-5

Dorn, A., Musilov'a, Z., Platzer, M., Reichwald, K., and Cellerino, A. (2014). The strange case of East African annual fishes: aridification correlates with diversification for a savannah aquatic group? BMC Evol. Biol. 14:210. doi: 10. 1186/s12862-014-0210-3

Dozawa, M., Kono, H., Sato, Y., Ito, Y., Tanaka, H., and Ohshima, T. (2014). Valproic acid, a histone deacetylase inhibitor, regulates cell proliferation in the adult zebrafish optic tectum. Dev. Dyn. 243, 1401-1415. doi: 10.1002/dvdy. 24173

Edelmann, K., Glashauser, L., Sprungala, S., Hesl, B., Fritschle, M., Ninkovic, J., et al. (2013). Increased radial glia quiescence, decreased reactivation upon injury and unaltered neuroblast behavior underlie decreased neurogenesis in the aging zebrafish telencephalon. J. Comp. Neurol. 521, 3099-3115. doi: 10. 1002/cne.23347

Fumagalli, M. R., Font-Clos, F., Milan, S., Zapperi, S., and La Porta, C. A. M. (2020). Comparative analysis of metabolic and transcriptomic features of Nothobranchius furzeri. J. R. Soc. Interface 17:20200217. doi: 10.1098/rsif.2020. 0217

Furness, A. I., Lee, K., and Reznick, D. N. (2015). Adaptation in a variable environment: phenotypic plasticity and bet-hedging during egg diapause and hatching in an annual killifish. Evolution 69, 1461-1475. doi: 10.1111/evo.12669

Genade, T., Benedetti, M., Terzibasi, E., Roncaglia, P., Valenzano, D. R., Cattaneo, A., et al. (2005). Annual fishes of the genus Nothobranchius as a model system for aging research. Aging Cell 4, 223-233. doi: 10.1111/j.1474-9726.2005.00165. $\mathrm{x}$

Grandel, H., Kaslin, J., Ganz, J., Wenzel, I., and Brand, M. (2006). Neural stem cells and neurogenesis in the adult zebrafish brain: origin, proliferation dynamics, migration and cell fate. Dev. Biol. 295, 263-277. doi: 10.1016/j.ydbio.2006.03. 040

Hand, S. C., Denlinger, D. L., Podrabsky, J. E., and Roy, R. (2016). Mechanisms of animal diapause: recent developments from nematodes, crustaceans, insects, and fish. Am. J. Physiol. Regul. Integr. Comp. Physiol. 310, R1193-R1211.

Harel, I., Benayoun, B. A., Machado, B. E., Priya Singh, P., Hu, C.-K., Pech, M. F., et al. (2015). A platform for rapid exploration of aging and diseases in a naturally short-lived vertebrate. Cell 160, 1013-1026. doi: 10.1016/j.cell.2015.01.038

Hartmann, N., and Englert, C. (2012). A microinjection protocol for the generation of transgenic killifish (Species: Nothobranchius furzeri). Dev. Dyn. 241, 11331141. doi: 10.1002/dvdy.23789

Hartmann, N., Reichwald, K., Lechel, A., Graf, M., Kirschner, J., Dorn, A., et al. (2009). Telomeres shorten while Tert expression increases during ageing of the short-lived fish Nothobranchius furzeri. Mech. Ageing Dev. 130, 290-296. doi: 10.1016/j.mad.2009.01.003

Hartmann, N., Reichwald, K., Wittig, I., Drose, S., Schmeisser, S., Luck, C., et al. (2011). Mitochondrial DNA copy number and function decrease with age in the short-lived fish Nothobranchius furzeri. Aging Cell 10, 824-831. doi: 10. 1111/j.1474-9726.2011.00723.x

Hu, C. K., Wang, W., Brind'Amour, J., Singh, P. P., Reeves, G. A., Lorincz, M. C., et al. (2020). Vertebrate diapause preserves organisms long term through Polycomb complex members. Science 367, 870-874. doi: 10.1126/ science.aaw2601

Ito, Y., Tanaka, H., Okamoto, H., and Ohshima, T. (2010). Characterization of neural stem cells and their progeny in the adult zebrafish optic tectum. Dev. Biol. 342, 26-38. doi: 10.1016/j.ydbio.2010.03.008

Kabiljo, J., Murko, C., Pusch, O., and Zupkovitz, G. (2019). Spatio-temporal expression profile of sirtuins during aging of the annual fish Nothobranchius furzeri. Gene Expr. Patterns 33, 11-19. doi: 10.1016/j.gep.2019.05.001

Kanungo, J., Goswami, M. T., and Pant, H. C. (2018). Notch and Cdk5 in Zebrafish Mindbomb Mutant: co-regulation or Coincidence?. Folia Biol. 64, 35-40.

Kaslin, J., Kroehne, V., Benato, F., Argenton, F., and Brand, M. (2013). Development and specification of cerebellar stem and progenitor cells in zebrafish: from embryo to adult. Neural Dev. 8:9. doi: 10.1186/1749-8104-8-9

Kelmer Sacramento, E., Kirkpatrick, J. M., Mazzetto, M., Baumgart, M., Bartolome, A., Di Sanzo, S., et al. (2020). Reduced proteasome activity in the aging brain results in ribosome stoichiometry loss and aggregation. Mol. Syst. Biol. 16:e9596. doi: 10.15252/msb.20209596

Kim, Y., Nam, H. G., and Valenzano, D. R. (2016). The short-lived African turquoise killifish: an emerging experimental model for ageing. Dis. Model. Mech. 9, 115-129. doi: 10.1242/dmm.023226

Knoth, R., Singec, I., Ditter, M., Pantazis, G., Capetian, P., Meyer, R. P., et al. (2010). Murine features of neurogenesis in the human hippocampus across the lifespan from 0 to 100 years. PLoS One 5:e8809. doi: 10.1371/journal.pone. 0008809<PMID<PMID:NOPMID</PMID<

Kuhn, H. G., Dickinson-Anson, H., and Gage, F. H. (1996). Neurogenesis in the dentate gyrus of the adult rat: age-related decrease of neuronal progenitor proliferation. J. Neurosci. 16, 2027-2033. doi: 10.1523/jneurosci.16-06-02027. 1996

Kuroyanagi, Y., Okuyama, T., Suehiro, Y., Imada, H., Shimada, A., Naruse, K., et al. (2010). Proliferation zones in adult medaka (Oryzias latipes) brain. Brain Res. 1323, 33-40. doi: 10.1016/j.brainres.2010.01.045

Love, M. I, Huber, W., and Anders, S. (2014). Moderated estimation of fold change and dispersion for RNA-seq data with DESeq2. Genome Biol. 15:550. doi: 10.1186/s13059-014-0550-8

Lugert, S., Basak, O., Knuckles, P., Haussler, U., Fabel, K., Gotz, M., et al. (2010). Quiescent and active hippocampal neural stem cells with distinct morphologies respond selectively to physiological and pathological stimuli and aging. Cell Stem Cell 6, 445-456. doi: 10.1016/j.stem.2010.03.017

Matsui, H., Kenmochi, N., and Namikawa, K. (2019). Age- and $\alpha$-SynucleinDependent Degeneration of Dopamine and Noradrenaline Neurons in the Annual Killifish Nothobranchius furzeri. Cell Rep. 26, 1727-1733.e6. doi: 10. 1016/j.celrep.2019.01.015

McIntosh, R., Norris, J., Clarke, J. D., and Alexandre, P. (2017). Spatial distribution and characterization of non-apical progenitors in the zebrafish embryo central nervous system. Open Biol. 7:160312. doi: 10.1098/rsob. 160312

Ming, G. L., and Song, H. (2011). Adult neurogenesis in the mammalian brain: significant answers and significant questions. Neuron 70, 687-702. doi: 10.1016/ j.neuron.2011.05.001

Montesano, A., De Felice, E., Leggieri, A., Palladino, A., Lucini, C., Scocco, P., et al. (2019). Ontogenetic Pattern Changes of Nucleobindin-2/Nesfatin-1 in the Brain and Intestinal Bulb of the Short Lived African Turquoise Killifish. J. Clin. Med. 9:103. doi: 10.3390/jcm9010103

Pekcec, A., Baumgartner, W., Bankstahl, J. P., Stein, V. M., and Potschka, H. (2008). Effect of aging on neurogenesis in the canine brain. Aging Cell 7, 368-374. doi: $10.1111 / j .1474-9726.2008 .00392 . x$

Pierfelice, T., Alberi, L., and Gaiano, N. (2011). Notch in the vertebrate nervous system: an old dog with new tricks. Neuron 69, 840-855. doi: 10.1016/j.neuron. 2011.02.031

Platzer, M., and Englert, C. (2016). Nothobranchius furzeri: a Model for Aging Research and More. Trends Genet. 32, 543-552. doi: 10.1016/j.tig.2016.06.006

Reichwald, K., Lauber, C., Nanda, I., Kirschner, J., Hartmann, N., Schories, S., et al. (2009). High tandem repeat content in the genome of the short-lived annual fish Nothobranchius furzeri: a new vertebrate model for aging research. Genome. Biol. 10:R16. doi: 10.1186/gb-2009-10-2-r16

Reichwald, K., Petzold, A., Koch, P., Downie, B. R., Hartmann, N., Pietsch, S., et al. (2015). Insights into Sex Chromosome Evolution and Aging from the Genome of a Short-Lived Fish. Cell 163, 1527-1538. doi: 10.1016/j.cell.2015. 10.071

Ripa, R., Dolfi, L., Terrigno, M., Pandolfini, L., Arcucci, V., Groth, M., et al. (2016). MicroRNA miR-29 controls a compensatory response to limit neuronal iron accumulation during adult life and aging. BMC Biol. 15:9. doi: 10.1186/s12915017-0354-x

Stankiewicz, A. J., McGowan, E. M., Yu, L., and Zhdanova, I. V. (2017). Impaired Sleep, Circadian Rhythms and Neurogenesis in Diet-Induced Premature Aging. Int. J. Mol. Sci. 18:2243. doi: 10.3390/ijms18112243

Sueda, R., and Kageyama, R. (2020). Regulation of active and quiescent somatic stem cells by Notch signaling. Dev. Growth Differ. 62, 59-66. doi: 10.1111/dgd. 12626

Suh, H., Deng, W., and Gage, F. H. (2009). Signaling in adult neurogenesis. Annu. Rev. Cell Dev. Biol. 25, 253-275.

Terzibasi, E., Valenzano, D. R., and Cellerino, A. (2007). The short-lived fish Nothobranchius furzeri as a new model system for aging studies. Exp. Gerontol. 42, 81-89. doi: 10.1016/j.exger.2006.06.039 
Terzibasi Tozzini, E., Dorn, A., Ng’oma, E., Polacik, M., Blazek, R., Reichwald, K., et al. (2013). Parallel evolution of senescence in annual fishes in response to extrinsic mortality. BMC Evol. Biol. 13:77. doi: 10.1186/1471-2148-13-77

Than-Trong, E., Ortica-Gatti, S., Mella, S., Nepal, C., Alunni, A., and BallyCuif, L. (2018). Neural stem cell quiescence and stemness are molecularly distinct outputs of the Notch 3 signalling cascade in the vertebrate adult brain. Development 145:dev161034. doi: 10.1242/dev.161034

Tozzini, E. T., Baumgart, M., Battistoni, G., and Cellerino, A. (2012). Adult neurogenesis in the short-lived teleost Nothobranchius furzeri: localization of neurogenic niches, molecular characterization and effects of aging. Aging Cell 11, 241-251. doi: 10.1111/j.1474-9726.2011.00781.x

Urbán, N., and Guillemot, F. (2014). Neurogenesis in the embryonic and adult brain: same regulators, different roles. Front Cell Neurosci. 8:396. doi: 10.3389/ fncel.2014.00396

Valenzano, D. R., Benayoun, B. A., Singh, P. P., Zhang, E., Etter, P. D., Hu, C. K., et al. (2015). The African turquoise killifish genome provides insights into evolution and genetic architecture of lifespan. Cell 163, 1539-1554. doi: 10.1016/j.cell.2015.11.008

Valenzano, D. R., Sharp, S., and Brunet, A. (2011). Transposon-Mediated Transgenesis in the Short-Lived African Killifish Nothobranchius furzeri, a Vertebrate Model for Aging. G3 1, 531-538. doi: 10.1534/g3.111.00 1271

Valenzano, D. R., Terzibasi, E., Cattaneo, A., Domenici, L., and Cellerino, A. (2006). Temperature affects longevity and age-related locomotor and cognitive decay in the short-lived fish Nothobranchius furzeri. Aging Cell 5, 275-278. doi: 10.1111/j.1474-9726.2006.00212.x

van Tetering, G., and Vooijs, M. (2011). Proteolytic cleavage of Notch: "HIT and RUN". Curr. Mol. Med. 11, 255-269. doi: 10.2174/156652411795677972

Vrtílek, M., Žák, J., Polačik, M., Blažek, R., and Reichard, M. (2018). Longitudinal demographic study of wild populations of African annual killifish. Sci. Rep. 8:4774. doi: 10.1038/s41598-018-22878-6
Wang, W., Hu, C. K., Zeng, A., Alegre, D., Hu, D., Gotting, K., et al. (2020). Changes in regeneration-responsive enhancers shape regenerative capacities in vertebrates. Science 369:eaaz3090. doi: 10.1126/science.aaz3090

Westin, J., and Lardelli, M. (1997). Three novel Notch genes in zebrafish: implications for vertebrate Notch gene evolution and function. Dev. Genes Evol. 207, 51-63. doi: 10.1007/s004270050091

Yeo, S. Y., Kim, M., Kim, H. S., Huh, T. L., and Chitnis, A. B. (2007). Fluorescent protein expression driven by her4 regulatory elements reveals the spatiotemporal pattern of Notch signaling in the nervous system of zebrafish embryos. Dev. Biol. 301, 555-567. doi: 10.1016/j.ydbio.2006. 10.020

Yoon, K., and Gaiano, N. (2005). Notch signaling in the mammalian central nervous system: insights from mouse mutants. Nat. Neurosci. 8, 709-715. doi: $10.1038 / \mathrm{nn} 1475$

Zupanc, G. K., and Horschke, I. (1995). Proliferation zones in the brain of adult gymnotiform fish: a quantitative mapping study. J. Comp. Neurol. 353, 213-233. doi: $10.1002 /$ cne. 903530205

Conflict of Interest: The authors declare that the research was conducted in the absence of any commercial or financial relationships that could be construed as a potential conflict of interest.

The reviewer, CL, declared a past collaboration with one of the authors, ETT, to the handling editor.

Copyright (c) 2021 Bagnoli and Terzibasi Tozzini. This is an open-access article distributed under the terms of the Creative Commons Attribution License (CC BY). The use, distribution or reproduction in other forums is permitted, provided the original author(s) and the copyright owner(s) are credited and that the original publication in this journal is cited, in accordance with accepted academic practice. No use, distribution or reproduction is permitted which does not comply with these terms. 